\title{
UX Research is Dead. Long live UX Research!
}

\author{
Stephen Giff \\ Microsoft Corp. \\ sgiff@microsoft.com
}

\author{
Huseyin Dogan \\ Bournemouth University \\ hdogan@bournemouth.ac.uk
}

\begin{abstract}
This paper critically discusses the future of User Experience (UX) research across the tech industry and hence the shift from classic usability research towards design-led approaches, quantitative research discipline, $A / B$ testing and telemetry. Likely causes for this shift are addressed to empower the UX research discipline to remain relevant. The areas covered include the identity crisis and democratization of UX research; evolvement and sustainability of the UX discipline; and fusion of UX across heterogeneous fields. The adaptations to this shift are discussed from the Point of View (PoV) of practitioners. This includes offering clarity and meaning based on the organizational context and product positioning; and also leveraging deep understanding human behavior with a focus on data integrity that relates to the technology and environment.
\end{abstract}

User Experience, UX Research, Design Thinking, UX Evolvement and Sustainability

\section{INTRODUCTION}

There has been a noticeable shift across the tech industry away from classic user experience (usability) research towards Design-led approaches, quantitative research, $A / B$ testing, and telemetry. This paper will take a look back at some likely causes for this shift, and then look forward to what can be done for the User Research discipline to remain relevant.

\section{THE BELLE-EPOQUE OF USER RESEARCH?}

At its peak, the UX Research discipline played a critical role in software organizations, whereby decisions were often not made until the product and its features had been tested with users typically in a make-shift, or newly constructed usability lab. As the discipline grew and became more highly ingrained into the product development cycle, the role of usability engineer flourished, and the User-Centred-Design movement was born (ISO-13407 1999). UX Researchers relied heavily on rigor and scientific method to present unbiased data, based primarily on Think-Aloud methodology (Lewis and Reiman 1993). Conducting research in this way however was costly, both in terms of money and time - particularly with the explosion of the web and the introduction of faster release cycles (Nielsen 1994). Discount methods were leveraged to take the insights/learning from years of practice, to efficiently kick the tires of a website or feature, and Rapid Iterative Testing and Evaluation (RITE) methods (Medlock et al. 2002) were also adopted to account for the emerging iterative nature of product development.

\subsection{From Tactics to Strategy}

Although the discipline had grown substantially, and the usability lab had become a security blanket for product teams, there was a feeling amongst researchers that this research was too tactical, and that in order to increase impact, it was imperative the discipline moved towards strategic/generative research. Many researchers were no longer running usability studies, instead they were conducting Ethnographic and Contextual Inquiry (Cl) Research, Interviews, Focus Groups, and Diary Studies. The strong desire in the industry to 'know our customers' brought persona development to the heart of the UCD movement (Cooper 1999). Being strategic however, takes time. Irreversible product decisions were often being made even before $\mathrm{Cl}$ interviews were complete. Although personas were all the rage, by overly focusing on audience characteristics, they seemed to solve a management rather than a design problem.

\subsection{Identity Crisis: Democratization of Research}

In the meantime, it was becoming increasingly difficult to make a distinction between UX and other research disciplines - like Market Research and 
Product Marketing. Furthermore, software organizations began executing user research without the need for UX researchers (Farooq and Munko 2015). UX Research had become one discipline among many that was conducting customer research, making it more and more difficult to be heard, to differentiate itself, and most importantly, to demonstrate a direct connection between its work and the product.

\subsection{Sea-Change: Agile, Design, and Data Science}

While the discipline arguably faced an apparent identity crisis, the Design and Data Science disciplines were emerging as principle players in a fast moving and agile product development culture. Design Thinking, $A B$ testing, data-modelling, and continuous integration/deployment further minimized the role of UX Research. Furthermore, the emergence of problem domains such as Data Science, Internet of Things and Cyber Security required a mind-set shift whereby, to ensure optimal impact, $\mathrm{HCl} / \mathrm{UX}$ practitioners are required to be embedded in multi- discipline teams.

\section{THE FUTURE: EVOLVING THE UX RESEARCH DISCIPLINE}

The $\mathrm{HCl} / \mathrm{UX}$ Research Discipline is relatively young, while the pace of technological innovation is rapid - as such it is natural that the UX Research discipline needs to evolve its methods and practices to meet this change. Farooq and Munko (2015) argued that the UX Research discipline is at an important inflection point, partly due to the democratization of UX Research, but also due to the software industry's need to explore user behaviour at scale. They further argue that in order to evolve, practitioners should:

(i) Uncover key human insights behind quantitative data

(ii) Strive to synthesize human behavioural insights to contribute to organizational knowledge of human insights over time

In addition to these critical recommendations, it is important to consider the following.

\subsection{Design Thinking: Design AND Research}

Design and Design Thinking is the responsibility of many disciplines including UX Research. The discipline plays a key role in uncovering human insights that ultimately lead to innovative and compelling solutions.

It is critical that academic institutions continue to encourage students to balance their left and right brains to ensure they ultimately move beyond preparing practitioners to uncover data and insights, instead towards encouraging them to use insights to identify innovative solutions in partnership with Designers, Technologists, and Business. Ultimately, UX Researchers need to ensure that they provide the bridge between uncovering human insights and solving real-world design problems.

\subsection{Democratization of Research: Embrace it!}

In small tech companies, there is often close alignment between research disciplines - however, in large companies, research can be silo'd, leading to duplication of efforts and a battle for the definitive truth. The ultimate goal of customer/user research is to uncover insights that will help create the most compelling experience for customers, and for these insights to be easily accessible to decision makers. Large tech companies should embrace the notion of 'one-research' and share research objectives/briefs, and insights with other disciplines, working with them to synthesize insights, and provide a single point of truth for their organization (Walter 2013). In addition, due to the cross-dimensional nature of the discipline, UX Researchers can play a key leadership role as curators of research data, helping to communicate the overall Point of View (POV) and play an important role of interpreting user needs in a language and format that business, leadership, engineering, and design understands.

\section{CONCLUSION: ADAPTING TO THE SHIFT}

Finally, although it is clear that the discipline needs to evolve to continue to remain relevant, it is critical for practitioners to remain true to the principles of the discipline by:

Focusing on people - this is at the heart of the discipline - by leveraging deep understanding of humans and human behavior - in particular as it relates to technology and the environment, together with a focus on data integrity - we are able to generate deep insights, and bring those insights to life, creating empathy and a connection between engineering and our customers.

Being strategic, with a product focus - choose projects and research questions wisely and be sure they align to the company goals/commitments. Uncover durable insights while iterating.

Establishing a POV - present a clear POV by taking a position on insights. Offer clarity and meaning based on the organizational context and product positioning.

Development and compilation of a new UX toolkit and strategies - centered on evolving disciplines such as Data Science, Internet of Things and Cyber Security. 


\section{REFERENCES}

Cooper, A. (1999) The inmates are running the asylum: Why high-tech products drive us crazy and how to restore the sanity, Indianapolis: Sams Publishing.

Farooq, U. and Munko, J.T. (2015) Industry Is Changing, and So Must We. In Proceedings of the 33rd Annual ACM Conference Extended Abstracts on Human Factors in Computing Systems. ACM, 655-661.

ISO 13407 (1999) Human-centred design processes for interactive systems. Geneva, Switzerland: International Organization for Standardization.
Lewis, C. Reiman, J. (1993) Task-Centered User Interface Design. A Practical Introduction. USA. Available from http://hcibib.org/tcuid/ (March 2016).

Medlock, M.C., Wixon, D., Terrano, M., Romero, R. and Fulton, B. (2002) Using the RITE method to improve products: A definition and a case study. Usability Professionals Association, 51.

Nielsen, J. (1994) Heuristic evaluation. Usability inspection methods, 17(1), 25-62.

Walter, A. (2013) Connected UX - A list apart. Available from http://alistapart.com/article/connected-ux (March 2016). 